\title{
Detailed S-Wave Velocity Structure of Sediment and Crust off Sanriku, Japan by a New Analysis Method for Distributed Acoustic Sensing Data Using a Seafloor Cable with Seismic Interferometry
}

Shun Fukushima ( $\sim$ s-fuku@eri.u-tokyo.ac.jp)

University of Tokyo: Tokyo Daigaku https://orcid.org/0000-0002-9522-9144

Masanao Shinohara

Earthquake Research Institute, University of Tokyo

Kiwamu Nishida

Earthquake Research Institute, University of Tokyo

Akiko Takeo

Earthquake Research Institute, University of Tokyo

Tomoaki Yamada

Earthquake Research Institute, University of Tokyo

Kiyoshi Yomogida

Hokkaido University of Graduate School of Science, Hokkaido University, Sapporo

\section{Research Article}

Keywords: S-wave velocity structure, Sediment and upper crust, Distributed acoustic sensing, Seismic interferometry, seafloor cable, Frequency wavenumber filter

Posted Date: February 23rd, 2022

DOI: https://doi.org/10.21203/rs.3.rs-1371937/v1

License: (c) (i) This work is licensed under a Creative Commons Attribution 4.0 International License.

Read Full License 
1 Detailed S-wave velocity structure of sediment and crust off Sanriku,

2 Japan by a new analysis method for distributed acoustic sensing data

3 using a seafloor cable with seismic interferometry

5 Author \#1: Shun Fukushima, Department of Earth and Planetary Science, Graduate

6 School of Science, The University of Tokyo, Tokyo, Japan, s-fuku@eri.u-tokyo.ac.jp

7 Author \#2: Masanao Shinohara, Earthquake Research Institute, University of

$8 \quad$ Tokyo, Tokyo, Japan, mshino@eri.u-tokyo.ac.jp

9 Author \#3: Kiwamu Nishida, Earthquake Research Institute, University of Tokyo,

10 Tokyo, Japan, knishida@eri.u-tokyo.ac.jp

11 Author \#4: Akiko Takeo, Earthquake Research Institute, University of Tokyo,

12 Tokyo, Japan, akiko-t@eri.u-tokyo.ac.jp

13 Author \#5: Tomoaki Yamada, Earthquake Research Institute, University of

14 Tokyo, Tokyo, Japan, yamada@eri.u-tokyo.ac.jp

15 Author \#6: Kiyoshi Yomogida, Department of Natural History Science, Graduate

16 School of Science, Hokkaido University, Sapporo, Japan, yomo@ sci.hokudai.ac.jp 
18 Indicate the corresponding author

19 Shun Fukushima

20 


\section{Abstract}

22 S-wave velocity $(V \mathrm{~s})$ structures of sediments and the uppermost crust in the landward

23 slope of a subduction zone are essential for elucidating the dynamics of the overriding

24 plate. Although distributed acoustic sensing (DAS) measurements have improved the

25 horizontal resolution of $V \mathrm{~s}$ structure in marine areas, the estimation has been limited in

26 the upper sedimental layer. This study imaged the structure from shallow to deep

27 sediments at a depth up to $7 \mathrm{~km}$ and the uppermost crust off Sanriku, Japan, with high

28 lateral resolution of $2.5 \mathrm{~km}$, by applying seismic interferometry to DAS data of 13 hours.

29 We grouped the DAS data into 10-km-long subarrays with $75 \%$ overlaps. We first applied

30 a frequency-wavenumber filter to the DAS data to remove DAS instrumental noise and

31 to effectively extract surface waves from short-time records. We then applied the seismic

32 interferometry method and estimated phase velocities at each subarray. The estimated

33 phase velocities of the fundamental- and first higher-mode Rayleigh waves were then

34 used to determine one-dimensional $V \mathrm{~s}$ structures for each subarray. The resultant 2-D $V \mathrm{~s}$

35 structure was interpreted as representing sediments and crust. The upper sediment layer

36 became thick seaward, and a discontinuity between sediment and crust layers at depths 
37 of 3-7 km showed strong lateral heterogeneities. By combining the result with the P-wave

38 velocity ( $V \mathrm{p})$ structure along the nearest survey line, we could estimate the $V \mathrm{p} / V \mathrm{~s}$ value

39 to be $2.28-4.0$ in the lowest sedimental layers. Our developed analysis method applying

40 seismic interferometry to marine DAS data can broaden the horizons for estimating

41 heterogeneous $V_{\mathrm{s}}$ and $V \mathrm{p} / V_{\mathrm{s}}$ structures of sediment layers and the upper crusts in

42 subduction zones. These results show that the application of both frequency-wavenumber

43 filtering and seismic interferometry to marine DAS data can be a new tool for estimating

44 the heterogeneous $V \mathrm{~s}$ and $V \mathrm{p} / V_{\mathrm{s}}$ structure of sediment layers and the upper crust in

45 subduction zones.

46

\section{Keywords}

48 S-wave velocity structure, Sediment and upper crust, Distributed acoustic sensing,

49 Seismic interferometry, seafloor cable, Frequency wavenumber filter

50 Main Text

\section{$51 \quad 1$ Introduction}

52 Accurate S-wave velocity structures $(V \mathrm{~s})$ of sediments and the uppermost crust in the 
53 landward slope of a subduction zone are indispensable information for elucidating the

54 dynamics of the overriding plate. $V$ s structures in a shallow region enhance the accuracy

55 of a wide range of studies, such as hypocenter determination and consideration of rock

56 properties. Since P-wave velocity structures $(V \mathrm{p})$ can be generally of high resolution,

57 reliable estimation of S-wave velocities should contribute to an accurate ratio of $\mathrm{P}$ - and

$58 \mathrm{~S}$-wave velocities $(\mathrm{Vp} / \mathrm{Vs})$, which is useful information for the estimation of rock

59 properties (Ayres and Theilen 1999).

60 In the subduction zone of the Japan trench, spatially high-resolution $V \mathrm{p}$ structures of

61 sediment and upper crust were obtained in space by the recent development of detailed

62 seismic surveys (Miura et al. 2003; Takahashi et al. 2004). However, the estimation of

$63 V$ s structures has been limited. Seismic surveys in the Japan trench could estimate Vs

64 structures based on clear recordings of $P$ to $S$ conversion waves (Fujie et al. 2018).

65 Although both seismic interferometry and receiver function methods are useful for

66 estimating Vs with ocean bottom seismometers (OBSs) records (Akuhara et al. 2020;

67 Yamaya et al. 2021; Yao et al. 2011), it has been difficult to obtain a high-resolution

68 spatial $V$ s model because the spatial density of observation stations in marine areas does 
69 not meet this quality.

70 In recent years, distributed acoustic sensing (DAS) measurements have been applied to

71 seismic observations (e.g., Zhan 2020). DAS measurements enable us to measure strain

72 velocities with spatially very high resolution over a long distance. The DAS

73 measurement is performed on both the land and seafloor. For example, Dou et al. (2017)

74 estimated shallow shear wave velocity structures by applying the ambient noise method

75 to DAS records on land. In a marine area, Spica et al. (2020) estimated the Vs structure

76 of shallow sediments (down to $3 \mathrm{~km}$ ) by applying the frequency-wavenumber (FK)

77 analysis to the seafloor DAS measurement obtained off the Sanriku coast of Japan.

78 However, their estimation of $V$ s structures was limited to a shallow portion of

79 sediments. It is necessary to obtain the $V$ s structure of all sediment and the upper crust

80 for the research of $V \mathrm{p} / V \mathrm{~s}$ ratios. The phase velocity of overtone or long period Rayleigh

81 waves is useful for estimating a deep part of the sediment and crust, since the phase

82 velocities of these waves are sensitive for $V s$ in the deep part.

83 This study applied a seismic interferometry method to seafloor DAS records to extract

84 overtone or long-period ( $>5 \mathrm{sec}$ ) Rayleigh waves. We also propose a practical method 
85 for extracting surface waves in a low-frequency range from short-time records with FK

86 filtering. We then estimated the phase velocities from the extracted Rayleigh waves.

87 Finally, we obtained a 2-D Vs structure model of sediment layers and the upper crust off

88 the Sanriku coast of northeast Japan.

\section{Data and Methods}

\section{$90 \quad 2.1$ Data}

91 In 1996, the Earthquake Research Institute of the University of Tokyo installed a

92 seafloor seismic tsunami observation system using an optical fiber cable at Sanriku

93 offshore (Figure 1). As the system contains six dark fibers, DAS measurement has been

94 conducted using a DAS interrogator unit of AP sensing (Cedilnik et al. 2019) since 14

95 February 2019. The data were recorded at a sampling frequency of $500 \mathrm{~Hz}$, and the

96 duration of the observations in this study was selected to be about 13 hours. The sensing

97 range, spatial resolution, and gauge length were $100 \mathrm{~km}, 5.1 \mathrm{~m}$, and $40.79 \mathrm{~m}$,

98 respectively. Shinohara et al. (2019) and Spica et al. (2020) described the cable setup

99 and measurement quality of this DAS system.

\subsection{Calculate CCFs with an FK filter}


101 Before calculating and stacking cross-correlation functions (CCFs) of DAS background

102 noise records, we divided the whole dataset of the DAS array into subarray ones (Figure

103 1). We decimated the original records by first reducing the sampling frequency from

$104500 \mathrm{~Hz}$ to $2 \mathrm{~Hz} ; 10$ adjacent data were then stacked to enhance signal-to-noise ratios

105 (SNRs) after filtering the anti-spatial aliasing. As a result, the spatial interval of the data

106 became $51 \mathrm{~m}$.

107 To compute CCFs, we first divided continuous records into 10-minute time window

108 segments. We allowed for a 50\% overlap of each segment to improve its SNR. We

109 computed the weighted average of the cross spectra between two channels, following

110 Takagi et al. (2021) and Takeo et al. (2013, 2014). This processing was conducted in the

111 frequency domain. To avoid any earthquake signals, we calculated the mean power

112 between 0.025 and $0.2 \mathrm{~Hz}$ in the frequency domain for each time window, and any

113 datasets whose amplitude exceeded 10 times the amplitude for the previous time

114 window were discarded. Takeo et al. (2013) provided more detailed explanations for the

115 calculation of CCFs.

116 In general, DAS instrumental noise has a coherent phase at the same time for all stations 
117 (Tribaldos and Franklin 2021). These noises particularly affect seismic interferometry

118 studies. In this study, we applied an FK filter to DAS data to remove this zero-lag noise

119 and enhance the SNR before calculating and stacking the CCFs. Given that the high

120 spatial density of DAS data enables us to apply spatial Fourier transformation, we

121 conducted a 2-D Fourier transformation in the FK domain. The zero-lag noise, which

122 has an infinity phase velocity, is mapped to the zero wavenumber in the FK domain. We

123 designed an FK filter $w(f, k)$ as:

124

$$
w(f, k)=g\left(f, k, c_{\min }\right) *\left(1-s\left(f, k, c_{\max }\right)\right)
$$

126

127 where $g\left(f, k, c_{\min }\right)$ is a $10 \%$ Tukey window to eliminate signals slower than the cut-

128 off minimum phase velocity $c_{\min } \cdot g\left(f, k, c_{\min }\right)$ is defined as:

129

$$
g\left(k, f, c_{\min }\right)
$$

$131=\left\{\begin{array}{lc}1, & \text { for }-0.4 k_{\max }<k<0.4 k_{\max } \\ 0, & \text { for } k<-0.5 k_{\max } \text { or } k>0.5 k_{\max } \\ \frac{1}{2}\left[1+\cos \left\{\pi\left(1-\frac{k}{0.1 k_{\max }}\right)\right\}\right], & \text { otherwise }\end{array}\right.$ 
133 where $k_{\max }=4 \pi f / c_{\min }$. Furthermore, $s\left(f, k, c_{\max }\right)$ is the 8-th order Kaiser filter to

134 remove the signal faster than the maximum phase velocity $c_{\max }$, including the zero-lag

135 noise. In this study, to enhance the surface wave signals, we set $c_{\min }$ and $c_{\max }$ to be

$136 \quad 0.35 \mathrm{~km} / \mathrm{s}$ and $4.0 \mathrm{~km} / \mathrm{s}$, respectively.

\section{$137 \quad 2.3$ Phase velocity estimation}

138 We adopted the spatial auto-correlation (SPAC) method to estimate phase velocity (Aki

139 1957; Okada 2006; Nishida et al. 2008b) because the SPAC method represents the

140 observed cross-spectra assuming a laterally homogeneous structure and a homogenous

141 source distribution. According to Nakahara et al. (2021), the synthetic cross-spectrum

142 for radial strain records (e.g., DAS) is defined as:

143

$144 S_{i j}^{\text {syn. }}\left(\omega, c_{\mathrm{R}}, c_{\mathrm{L}}\right)=A_{\mathrm{R}}(\omega)\left[3 J_{0}\left(\frac{\omega}{c_{\mathrm{R}}} d_{i j}\right)-4 J_{2}\left(\frac{\omega}{c_{\mathrm{R}}} d_{i j}\right)+J_{4}\left(\frac{\omega}{c_{\mathrm{R}}} d_{i j}\right)\right]+B_{\mathrm{L}}(\omega)\left[J_{0}\left(\frac{\omega}{c_{\mathrm{L}}} d_{i j}\right)-J_{4}\left(\frac{\omega}{c_{\mathrm{L}}} d_{i j}\right)\right]$,

146 Where $\omega$ is the angular frequency, $A_{\mathrm{R}}$ is the power spectrum for Rayleigh wave, $B_{\mathrm{L}}$

147 is the power spectrum for Love wave, $J_{n}$ is the nth-order Bessel function of the first 
148 kind, $d_{i j}$ is the distance between $i$ th and $j$ th channels, $c_{\mathrm{R}}$ is the phase velocity of the

149 Rayleigh wave, and $c_{\mathrm{L}}$ is the phase velocity of Love wave. In Equation 3, the first and

150 second terms represent the Rayleigh and Love waves, respectively. In general, Love

151 waves have less energy than Rayleigh waves at a period range below $0.1 \mathrm{~Hz}$ (Nishida et

152 al. 2008a). Furthermore, as mentioned by Nakahara et al. (2021), Rayleigh waves

153 predominate in the far field. The envelope of the Rayleigh waves term decays with an

154 order of $d^{-1 / 2}$, while Love-wave term decays with an order of $d^{-3 / 2}$ in the far field.

155 For these reasons and given the small, estimated contribution of the Love wave terms,

156 we therefore obtained our misfit function $E$ as:

157

$$
\begin{gathered}
L 2\left(\omega, c_{\mathrm{R}}\right)=\sum_{i j}\left(\operatorname{Re}\left[S_{i j}^{\mathrm{obs}}(\omega)\right]-S_{i j}^{\mathrm{syn} .}\left(\omega, c_{\mathrm{R}}\right)\right)^{2}, \\
E\left(\beta_{l}, h_{l}\right)=\frac{1}{\omega} \int L 2\left(\omega, c_{R}\left(\omega ; \beta_{l} ; h_{l}\right)\right) d \omega,
\end{gathered}
$$

160

161 where $S_{i j}^{\mathrm{obs}}(\omega)$ and $S_{i j}^{\mathrm{syn} .}(\omega)$ are the observed and synthetic cross-spectra between $i$ th 162 and $j$ th channels calculated from the equation 3 , respectively. $\beta_{l}$ and $h_{l}$ are the $V$ s and 
164 and Yoshizawa \& Kennett. (2002) to estimate the phase velocity model. $\beta_{l}$ and $h_{l}$ are

165 assumed to be model parameters for minimizing $E\left(\beta_{l}, h_{l}\right)$ in Equation 5 by smoothing

166 the dispersion curve. To search for the sets of model parameters for minimizing the

167 value of the misfit function, we adopted the simulated annealing algorithm of Goffe et

168 al. (1994) as a global optimizer for searching for the minimum value of Equation 5. To

169 avoid numerical instability, we constrained the $V \mathrm{~s}$ at each layer, which was greater than

$17080 \%$ of that of the layer directly above.

171 In practice, we estimated the phase velocity and evaluated the errors in the estimated

172 phase velocity. First, we estimated the dispersion curves of phase velocity from cross-

173 spectra for all pairs of channels for each subarray. We then obtained a 1-D model with

174 six layers, where the deepest layer had infinite thickness for each subarray. We adopted

175 the scaling relationship between $V \mathrm{p}, V \mathrm{~s}$, and density from Brocher's (2005) results.

176 Next, searching by trial and error, we divided the subarrays into two groups according

177 to the distances from the coast. The dispersion curves of the phase velocity were

178 estimated using the fundamental mode of the Rayleigh wave only for one group, which

179 has distances ranging from $35 \mathrm{~km}$ to $50 \mathrm{~km}$ from the coast. For another group with 
180 distances ranging from $50 \mathrm{~km}$ to $75 \mathrm{~km}$, the fundamental mode and the first higher

181 mode of Rayleigh wave were used to obtain a dispersion curve. Synthetic phase

182 velocities were calculated using DISPER80 (Saito 1988).

183 Furthermore, we used the bootstrap method (Efron 1992) to estimate errors. We

184 aggregated station pairs randomly selected from all station pairs, allowing for overlap

185 for a bootstrap sample. Here, we calculated the standard deviation of 100 dispersion

186 curves estimated from each of the 100 bootstrap samples. The Vs structures estimated

187 from the data of all pairs of channels for each subarray were used as the reference

188 model.

189

$190 \quad 2.4$ Inversion of the S-wave velocity structure

191 From the phase velocity of the Rayleigh wave estimated by seismic interferometry, a 1-

$192 \mathrm{D} V \mathrm{~s}$ isotropic model was obtained. The 1-D Vs structure was determined by

193 minimizing the misfit function $E$, as defined by:

194

195

$$
E=\sqrt{\frac{1}{N} \Sigma\left[\frac{c_{o b s}(\omega)-c_{s y n}\left(\omega ; \beta_{l} ; h_{l}\right)}{c_{e r r}(\omega)}\right]^{2}}
$$


197 where $N$ is the number of estimated phase velocities. $c_{o b s}, c_{s y n}$ and $c_{\text {err }}$ indicate the

198 observed, synthesized, and uncertainty of the phase velocity, respectively. To obtain a

199 stable result, only a frequency band with phase velocities with an error lower than 0.1

$200 \mathrm{~km} / \mathrm{s}$ was used for the inversion analysis. We again set the $V \mathrm{~s}\left(\beta_{l}\right)$ and thickness $\left(h_{l}\right)$

201 as the model parameters to minimize Equation 6. We then used the simulated annealing

202 algorithm method to obtain the optimal model parameters. The bootstrap average

203 models estimated from the phase velocity estimation were used as the reference model.

204 To estimate errors, we used the bootstrap method once again. Here, we aggregated the

205 final dispersion curve randomly selected from the 100 dispersion curves estimated in

206 the previous section (Takeo et al. 2013).

\section{Results and Discussion}

208 We computed the CCFs between all possible pairs of each subarray. The CCFs clearly

209 showed a surface wave (Figure 2). The group velocities of about $0.5 \mathrm{~km} / \mathrm{s}$ were much

210 slower than the surface waves in land observations in the same period range. The

211 surface wave with low velocity corresponded to a special type of Rayleigh wave or 
212 Scholte wave (Hovem et al. 2012; Bagheri et al. 2015) because the Love wave had less

213 energy than the Rayleigh wave in the period range below $0.1 \mathrm{~Hz}$. The CCFs with the FK

214 filter had a much higher SNR than those without the FK filter. For the estimation of

215 SNR, the method of Bensen et al. (2007) was used.

216 We selected the dispersion curve from the CFFs (Figures 3-A and 3-E). Extracting

217 Rayleigh waves by interferometry enabled us to stably estimate dispersion curves with

218 errors less than $0.1 \mathrm{~km} / \mathrm{s}$ by bootstrap. Near the coast (Figure 3-A), the fundamental

219 Rayleigh wave was dominant in the frequency range between 0.20 and $0.5 \mathrm{~Hz}$. By

220 contrast, in the case of an offshore subarray (Figure 3-E), both fundamental (0.5-0.1

$221 \mathrm{~Hz})$ and 1st higher mode $(0.5-0.2 \mathrm{~Hz})$ Rayleigh waves were dominant. This result

222 clearly indicates that the thickness of the low- $V$ s layer differs between near-coast and

223 offshore areas. We determined the $V$ s structure using only the phase velocities of the

224 fundamental mode of Rayleigh waves in the near-coast area at distances between $30 \mathrm{~km}$

225 and $50 \mathrm{~km}$. In the area where the distance was greater than $50 \mathrm{~km}$ from the coast, both

226 the fundamental and first higher modes of the Rayleigh waves were used for phase

227 velocity estimation. 
228 We inverted the measured phase velocities into 1-D Vs structures (Figure 3) below each

229 subarray. The 1-D Vs structures (Figures 3-B and 3-F) and normalized Vs sensitivity

230 kernels (Figures 3-C, 3-G, and 3-I) of the Rayleigh waves were calculated. Errors were

231 calculated using the bootstrap method. A small error in the depth range, whose Vs

232 values were slower than $2.0 \mathrm{~km} / \mathrm{s}$ were estimated. Synthetic phase velocities calculated

233 by DISPER80 using the 1-D Vs inversion results were consistent with the observations

234 (Figures 3-D and 3-H). Given that the sensitivity kernel had high sensitivity in the depth

235 range of $V \mathrm{~s}$ slower than $2.0 \mathrm{~km} / \mathrm{s}$, our inversion provided a $V \mathrm{~s}$ structure with small

236 errors at the depth range of $V \mathrm{~s}$ slower than $2.0 \mathrm{~km} / \mathrm{s}$. By contrast, layers of $V \mathrm{~s}$ greater

237 than $2.0 \mathrm{~km} / \mathrm{s}$ have uncertainty. However, the $V \mathrm{~s}$ sharply increases to greater than 2.0

$238 \mathrm{~km} / \mathrm{s}$ at the boundaries, and the error range for the lowermost layer does not overlap

239 with that of the upper layers. Nevertheless, the depth of discontinuity between the

240 lowermost layer and the upper layer was estimated with high resolution.

241 Finally, a 2-D Vs structure model (Figure 4) was obtained from the 1-D Vs structures at

242 each subarray. The 2-D structure model was composed of four layers. The $V$ s of the first

243 and second layers at depths of $0-1 \mathrm{~km}$ below the sea floor were slower than $0.6 \mathrm{~km} / \mathrm{s}$ 
244 and approximately $0.7 \mathrm{~km} / \mathrm{s}$, respectively. The $V s$ of the third and fourth layers at depths

245 of $2-6 \mathrm{~km}$ below the sea floor were $1.75 \mathrm{~km} / \mathrm{s}$ and greater than $2.0 \mathrm{~km} / \mathrm{s}$, respectively.

246 We compared our 2-D Vs structure (Figure 4) with the Vs and Vp structures obtained by

247 other studies to interpret these layers. The $V$ s values of the first and second layers were

248 smaller than $0.6 \mathrm{~km} / \mathrm{s}$ and about $0.7 \mathrm{~km} / \mathrm{s}$, respectively, and the velocities are consistent

249 with the results of a study by Spica et al. (2020). Takahashi et al. (2004) provided the

$250 V \mathrm{p}$ structure estimated by refraction surveys along a profile parallel to our DAS cable.

251 Takahashi et al.'s (2004) profile was positioned northward and had a distance of about

$25220 \mathrm{~km}$ from the seafloor cable. The $V \mathrm{p}$ of the first and second layers were determined as

2531.7 and $2.3 \mathrm{~km} / \mathrm{s}$, respectively, and the layers were interpreted as Neogene sediment by

254 Takahashi et al. (2004), Kodaira et al. (2017), and Spica et al. (2020). The thickness of

255 the first and second layers increased seaward in a distance range from $30 \mathrm{~km}$ to $55 \mathrm{~km}$.

256 This increase in thickness is also consistent with the results of a study by Spica et al.

257 (2020). However, the thickness of the first and second layers showed no large variation

258 in the distance range between 55 and $80 \mathrm{~km}$.

259 The third layer was thick $(\sim 1$ to $\sim 5 \mathrm{~km})$ in the distance range from the coast from 30 to 
$26055 \mathrm{~km}$. However, the thickness of the third layer was small ( $\sim 5$ to $\sim 3 \mathrm{~km})$ in a distance

261 range from 55 to $65 \mathrm{~km}$. At distances between $65 \mathrm{~km}$ and $75 \mathrm{~km}$, the third layer

262 disappeared. These lateral heterogeneities of the third layer were also shown in the $\mathrm{Vp}$

263 model by Takahashi et al. (2004). The discontinuity between the third and fourth layers

264 can be interpreted as the boundary between sediments and the island arc uppermost

265 crust because the depth of the interface between the third layer and the fourth layer is

266 consistent with that reported by Takahashi et al. (2004). Below the landward slope off

267 the Japan trench at Tohoku, it is known that the sediments are large lateral

268 heterogeneous layers perpendicular to the Japan trench. (Ito et al. 2004; Takahashi et al.

269 2004).

270 Although our profile and the profile of Takahashi et al. (2004) are $20 \mathrm{~km}$ apart, we

271 estimated the $V \mathrm{p} / V \mathrm{~s}$ ratio of the third layer. Given that $V \mathrm{~s}$ was $1.1-1.75 \mathrm{~km} / \mathrm{s}$ from our

272 results, and $V \mathrm{p}$ was estimated to be $4.0-4.4 \mathrm{~km} / \mathrm{s}$ by Takahashi et al. (2004), $V \mathrm{p} / \mathrm{Vs}$ ratio

273 in the third layer would be $2.28-4.0$. However, $V \mathrm{p} / V \mathrm{~s}$ ratio of the fourth layer was

274 smaller than 2.5 because $V \mathrm{~s}$ and $V \mathrm{p}$ of the fourth layer were faster than 2.0 and $5.0 \mathrm{~km} / \mathrm{s}$,

275 respectively. Fujie et al. (2018) estimated that the Vp/Vs ratio of the oceanic crust was 
lower than 1.9 by seismic refraction. By contrast, Kodaira et al. (1996) estimated the

$277 \quad V \mathrm{p} / \mathrm{Vs}$ value of the upper crust to be $1.67-1.78$ at the Lofoten passive continental margin

278 in northern Norway. The estimated $V \mathrm{p} / V \mathrm{~s}$ value of the third layer in our study was

279 higher than their results for oceanic igneous crusts. However, Chian and Louden (1994)

280 showed a $V \mathrm{p} / V \mathrm{~s}$ ratio of 4.0 for the sediment layer in south-western Greenland, and

281 Kodaira et al. (1996) found the $V \mathrm{p} / V \mathrm{~s}$ value of the sediment layers to be 3.0-5.0 in

282 northern Norway. As described by Kodaira et al. (1996), unconsolidation is most

283 effective for the large $V \mathrm{p} / V \mathrm{~s}$ ratio of sediment layers. Some aftershocks of the 2011

284 Tohoku-oki earthquake occurred in the fourth layer (Figure 4), whereas there was no

285 seismicity in the upper layers. As a result, the third layer can be interpreted as a

286 sediment layer, and the interface between the third layer and the fourth layer represents

287 the upper surface of the igneous crust.

288 The estimation of $V$ s structures using DAS records in previous studies has been limited

289 to a shallow part of crusts. Spica et al. (2020) estimated the Vs structure to be up to 3

$290 \mathrm{~km}$ in depth. The seismic interferometry with FK filtering to DAS data enables the

291 determination of phase velocities of surface waves whose periods are longer than those 
292 of previous studies. Therefore, we could obtain an S-wave structure in depth up to $8 \mathrm{~km}$

293 using the DAS data with high spatial resolution. Yamaya et al. (2021) estimated a three-

294 dimensional $V$ s structure using data from an OBS network with an interval distance of 6

$295 \mathrm{~km}$ off Ibaraki, Japan, by a tomography method. Generally, the resolution of seismic

296 tomography depends on the spatial density of the stations. By contrast, the spatial

297 horizontal resolution of the 2-D Vs structure in this study is believed to be higher due to

298 the high spatial density of the observation. Vs structures in oceanic crusts have usually

299 been estimated by observation of $P$ to $S$ conversion waves during seismic surveys or

300 seismic interferometry using spatially dense OBSs data. Our method offers a new

301 approach to determine the $V$ s of shallow structures with high spatial resolution in

302 marine areas from short-term DAS data using a seafloor cable. A shallow Vs structure in

303 a marine area with high resolution by our method leads to a detailed distribution of

$304 \mathrm{Vp} / V_{\mathrm{s}}$ ratio in a shallow region, which is useful for considering tectonics and rock

305 properties.

\section{$306 \quad 5$ Conclusions}

307 The information of $V \mathrm{~s}$ is important to understand the rock properties of the upper crust 
and sediments on the seafloor. We applied the seismic interferometry method to DAS records with a recording period of 13 hours by seafloor cable installed off Sanriku,

310 Japan. Applying the FK filter to the DAS data before calculating the CCFs effectively

311 enhances surface waves. The phase velocities of the Rayleigh waves were calculated by

312 the SPAC method. Finally, we inverted the $V$ s structure from the phase velocities of the

313 Rayleigh waves obtained from seismic interferometry. The $V$ s structures of the sediment

314 layers and the upper crust were consistent with those determined by refraction surveys.

315 The seismic interferometry method with DAS data collected through seafloor cables

316 could estimate $V$ s structures with high spatial resolution.

317 To explore the $V$ s structure of sediment layers, seismic interferometry and receiver

318 function methods have been widely conducted using OBS data. However, their spatial

319 resolution is constrained by the spatial density of the installed OBSs. By contrast, a Vs

320 structure with high spatial resolution can be estimated from short-term DAS records by

321 applying the seismic interferometry method. Our results showed a new approach to

322 estimating the heterogeneous $V$ s structure of sediment layers and the upper crust in

323 subduction zones. 


\section{Declarations}

327

328

329

330

331

332

333

334

335

336

337

338

339

340
Ethics approval and consent to participate

Not applicable.

\section{Consent for publication}

Not applicable.

\section{List of abbreviations}

$V_{\mathrm{S}}: \mathrm{S}$-wave velocity

$V_{\mathrm{P}}: \mathrm{P}$-wave velocity

$V \mathrm{p} / V \mathrm{~s}$ : ratio of $\mathrm{P}$ - and $\mathrm{S}$-wave velocities

DAS: distributed acoustic sensing

FK: frequency-wavenumber

CCFs : cross-correlation functions

SNRs : signal-to-noise ratios

SPAC : spatial auto-correlation

OBSs : ocean bottom seismometers 


\section{Availability of data and materials}

343

The DAS observations were performed as part of the Earthquake and

Volcano Hazards Observation and Research Program (Earthquake and

Volcano Hazard Reduction Research) by the Ministry of Education,

Culture, Sports, Science and Technology of Japan. These data are

available from the corresponding author on request.

\section{Competing interests}

The authors acknowledge that there are no conflicts of interest recorded.

This study was mainly supported by the Ministry of Education, Culture, 
SF played a leading role in this study, including data processing,

All authors read and approved the final manuscript.

\section{Acknowledgements} and Messrs. T. Hashimoto, T. Yagi of the Earthquake Research Institute, the University of Tokyo for technical support of the DAS observation. The collaboration with Fujitsu Laboratories Ltd. in the field of data acquisition is appreciated. Discussion with Drs. Akuhara and Takemura was fruitful for 


\section{Authors' information}

376
Shun Fukushima ${ }^{1,2}$, Masanao Shinohara ${ }^{2}$, Kiwamu Nishida ${ }^{2}$, Akiko

Takeo $^{2}$, Tomoaki Yamada ${ }^{2}$, and Kiyoshi Yomogida ${ }^{3}$

\footnotetext{
${ }^{1}$ Department of Earth and Planetary Science, Graduate School of
}

Science, The University of Tokyo, 7-3-1 Hongo, Bunkyo-ku, Tokyo,

113-0033, Japan

${ }^{2}$ Earthquake Research Institute, The University of Tokyo, 1-1-1, Yayoi, Bunkyo-ku, Tokyo, 113-0032, Japan

${ }^{3}$ Department of Natural History Science, Graduate School of Science, Hokkaido University, Kita10 nishi-8 Kita-ku, Sapporo, Hokkaido 0600810, Japan

\section{References}


Aki K (1957) Space and time spectra of stationary stochastic waves, with special reference to microtremors. Bulletin of the Earthquake Research Institute $35: 415-456$

Akuhara T, Tsuji T, Tonegawa T (2020) Overpressured Underthrust Sediment in the Nankai Trough Forearc Inferred From Transdimensional Inversion of HighFrequency Teleseismic Waveforms. Geophysical Research Letters 47: https://doi.org/10.1029/2020GL088280

Ayres A, Theilen F (1999) Relationship between P-and S-wave velocities and geological properties of near-surface sediments of the continental slope of the Barents Sea. Geophysical prospecting 47:431-441 Scholte, Stoneley and Love waves in a model consisting of a liquid layer overlying a two-layer transversely isotropic solid medium. Geophysical Journal International 203:195-212. https://doi.org/10.1093/gji/ggv278 

acoustic sensing for power cable monitoring. In: Proceedings of the JICABLE Chian D, Louden KE (1994) The continent-ocean crustal transition across the southwest Greenland margin. Journal of Geophysical Research: Solid Earth 99:9117-9135 Study. Scientific Reports 7:. https://doi.org/10.1038/s41598-017-11986-4 Efron B (1992) Bootstrap methods: another look at the jackknife. In: Breakthroughs in statistics. Springer, pp 569-593 Fujie G, Kodaira S, Kaiho Y, et al (2018) Controlling factor of incoming plate https://doi.org/10.1038/s41467-018-06320-z 
Hovem JM, Richardson MD, Stoll RD (2012) Shear waves in marine sediments.

Hunter JD (2007) Matplotlib: A 2D graphics environment. Computing in Science \& 1994 Sanriku-oki earthquake. Earth and Planetary Science Letters 223:163-175. https://doi.org/10.1016/j.eps1.2004.04.007 Geophysical Journal International 124:724-740 characters in the rupture zone of the 2011 Tohoku-oki earthquake. Geosphere 13:1408-1424. https://doi.org/10.1130/GES01489.1 the seiscimity of the southern Japan Trench fore-arc region, revealed by ocean bottom seismographic data. Tectonophysics 363:79-102. 
Nakahara H, Emoto K, Nishimura T (2021) Extending the formulation of the spatial autocorrelation (SPAC) method to strain, rotation and tilt. Geophysical Journal International 227:287-302 waves simultaneously generated at the Pacific Ocean floors. Geophysical Research Letters 35:

Nishida K, Kawakatsu H, Obara K (2008b) Three-dimensional crustal S wave velocity structure in Japan using microseismic data recorded by Hi-net tiltmeters. Journal of Geophysical Research: Solid Earth 113:

Okada H (2006) Theory of efficient array observations of microtremors with special reference to the SPAC method. Exploration Geophysics 37:73-85. https://doi.org/10.1071/EG06073 ambient seismic noise recorded with distributed acoustic sensing (DAS) 
Saito M (1988) DISPER80: A subroutine package for the calculation of seismic normal-mode solutions. Seismological algorithms 293-319 measurement by using seafloor optical fiber cable system off Sanriku for seismic observation. In: OCEANS 2019 MTS/IEEE SEATTLE. pp 1-4 https://doi.org/10.1029/2020GL088360

Takagi R, Toyokuni G, Chikasada N (2021) Ambient noise correlation analysis of S-

Spica ZJ, Nishida K, Akuhara T, et al (2020) Marine Sediment Characterized by Ocean-Bottom Fiber-Optic Seismology. Geophysical Research Letters 47:. Geophysical Journal International 224:1640-1657 off Sanriku region, northeastern Japan. Geophysical Journal International 159:129-145. https://doi.org/10.1111/j.1365-246X.2004.02350.x 
anisotropy in the NW Pacific from seismic ambient noise in seafloor records. Shikoku Basin from broadband surface wave analysis of ocean bottom seismometer records. Journal of Geophysical Research: Solid Earth 118:28782892. https://doi.org/10.1002/jgrb.50219

A. Takeo, K. Nishida, H. Aoyama, M. Ishise, T. Kai, R. Kurihara, T. Maeda, Y. (2022), S-wave modeling of the Showa-Shinzan lava dome in Usu Volcano, Geochemistry, Geophysics, Geosystems 20:5556-5564 Northern Japan, from seismic observations, EarthArXiv, doi:10.31223/X5GW5M.

Wessel P, Luis JF, Uieda L, et al (2019) The generic mapping tools version 6 . 

lithosphere from ambient noise correlation analysis of fundamental- and higher-mode Scholte-Rayleigh waves. Comptes Rendus - Geoscience 343:571583. https://doi.org/10.1016/j.crte.2011.04.004 Yoshizawa K, Kennett BLN (2002) Non-linear waveform inversion for surface https://doi.org/10.1046/j.1365-246X.2002.01634.x Zhan Z (2020) Distributed acoustic sensing turns fiber-optic cables into sensitive seismic antennas. Seismological Research Letters 91:1-15

\section{$501 \quad$ Figure 1}

502 Short title : Location of the seafloor cable containing optical fibers in the off-Sanriku 
503 region, Japan.

\section{Detailed legend}

505 Location of the seafloor cable containing optical fibers in the off-Sanriku region, Japan.

506 The black line indicates the seafloor cable installed by the Earthquake Research

507 Institute, the University of Tokyo. The red dots indicate the center locations of the

508 subarrays to calculate the CCFs in this study.

509

$510 \quad$ Figure 2

511 Short title: Calculated CCFs.

\section{Detailed legend:}

513 Calculated CCFs. Each trace was normalized by its amplitude. (A) and (B) - CCFs at a

514 distance of $39.94 \mathrm{~km}$ from the coast without and with an FK filter, respectively. The

515 frequency range was between 0.17 and $0.50 \mathrm{~Hz}$. (C) and (D) - CCFs without and with

516 an FK filter at $59.96 \mathrm{~km}$ from the coast in the frequency range between 0.10 and 0.25

517 Hz. SNR indicates the signal-to-noise ratios calculated by Bensen's (2007) method. The

518 dashed lines indicate an apparent velocity of $0.5 \mathrm{~km} / \mathrm{s}$. The FK filter improves SNR 
519 values and enables us to extract surface waves effectively.

520

$521 \quad$ Figure 3:

522 Short title: Phase velocity, Vs structures, and sensitivity kernel.

\section{Detailed legend:}

524 (A) and (E) - Normalize residuals and estimation phase velocity at subarrays from the

525 coast of 32.43 and $67.68 \mathrm{~km}$, respectively. The error bars represent the uncertainties

526 estimated by the bootstrap method. The white dots and cross makers indicate errors less

527 than and more than $0.1 \mathrm{~km} / \mathrm{s}$, respectively. We used phase velocity only with an error

528 less than $0.1 \mathrm{~km} / \mathrm{s}$ for our inversion analysis. (B) and (F) - Vs structures at subarrays

529 from the coast of 32.43 and $67.68 \mathrm{~km}$. (D) and (H) - The white dots and green lines

530 indicate the observed and estimated phase velocities. The errors were estimated by the

531 bootstrap method. (C) - The normalized Vs sensitivity kernel of the fundamental

532 Rayleigh wave at $32.43 \mathrm{~km}$ from the coast. (G) and (I) - The normalized $V$ s sensitivity

533 kernels of fundamental and first higher mode Rayleigh waves at $67.68 \mathrm{~km}$ from the

534 coast. 


\section{$536 \quad$ Figure 4:}

537 Short title: 2-D Vs structure estimated from phase velocity information.

\section{Detailed legend:}

539 2-D Vs structure estimated from phase velocity information. The white circles indicate

540 the aftershocks off the Pacific coast of the Tohoku Earthquake determined by pop-up

541 OBSs (Shinohara et al., 2012). The aftershocks within a box of $40 \mathrm{~km}$ in width on both

542 sides along the fiber cable are projected onto this profile.

543

\section{Preparing additional files}

545 Additional figures.

546 
Figures

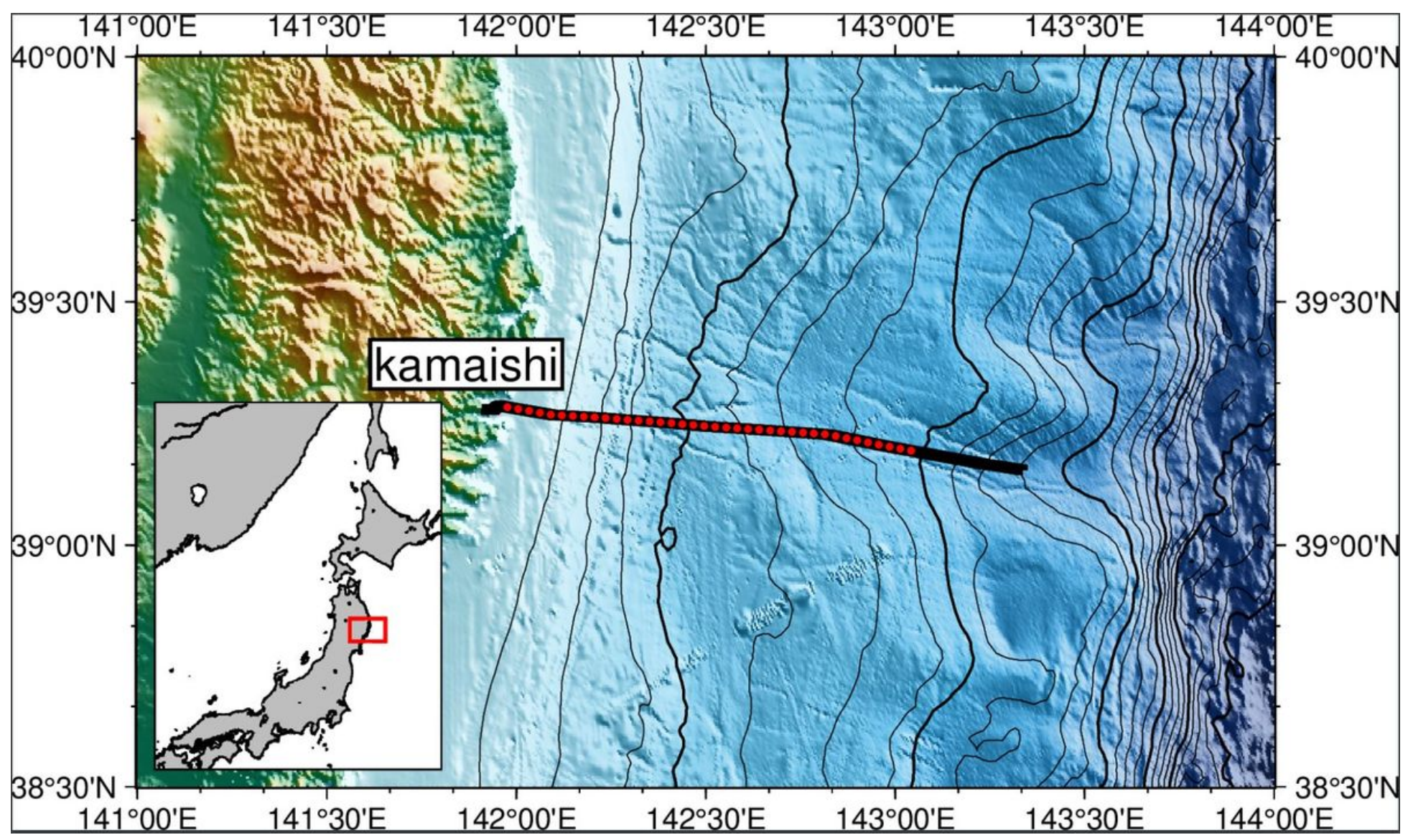

Figure 1

Short title : Location of the seafloor cable containing optical fibers in the off-Sanriku region, Japan.

\section{Detailed legend}

Location of the seafloor cable containing optical fibers in the off-Sanriku region, Japan. The black line indicates the seafloor cable installed by the Earthquake Research Institute, the University of Tokyo. The red dots indicate the center locations of the subarrays to calculate the CCFs in this study. 

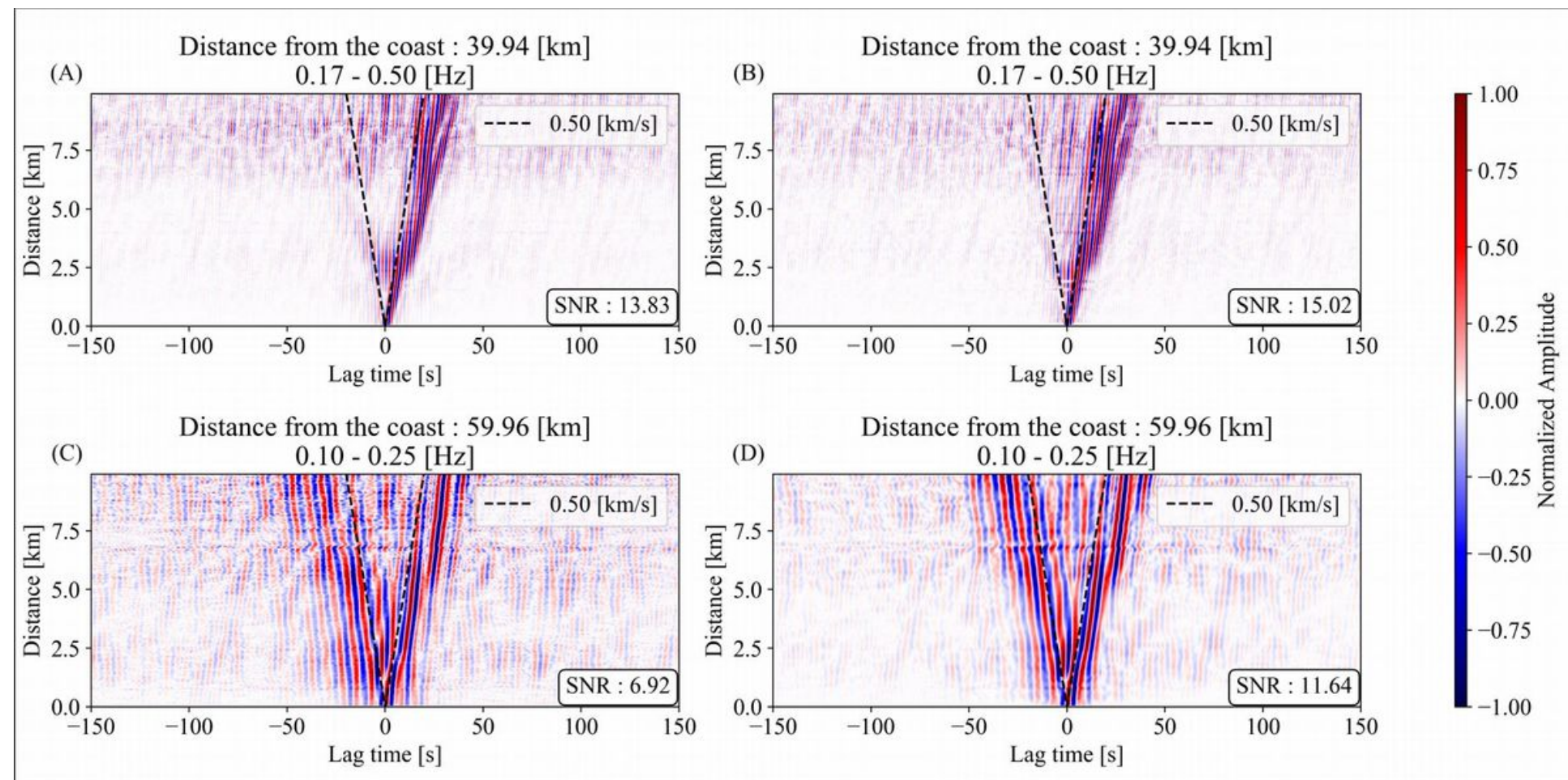

Figure 2

Short title: Calculated CCFs.

Detailed legend:

Calculated CCFs. Each trace was normalized by its amplitude. (A) and (B) - CCFs at a distance of 39.94 $\mathrm{km}$ from the coast without and with an FK filter, respectively. The frequency range was between 0.17 and $0.50 \mathrm{~Hz}$. (C) and (D) - CCFs without and with an FK filter at $59.96 \mathrm{~km}$ from the coast in the frequency range between 0.10 and $0.25 \mathrm{~Hz}$. SNR indicates the signal-to-noise ratios calculated by Bensen's (2007) method. The dashed lines indicate an apparent velocity of $0.5 \mathrm{~km} / \mathrm{s}$. The FK filter improves SNR values and enables us to extract surface waves effectively. 
Distance from the coast : $32.43[\mathrm{~km}]$

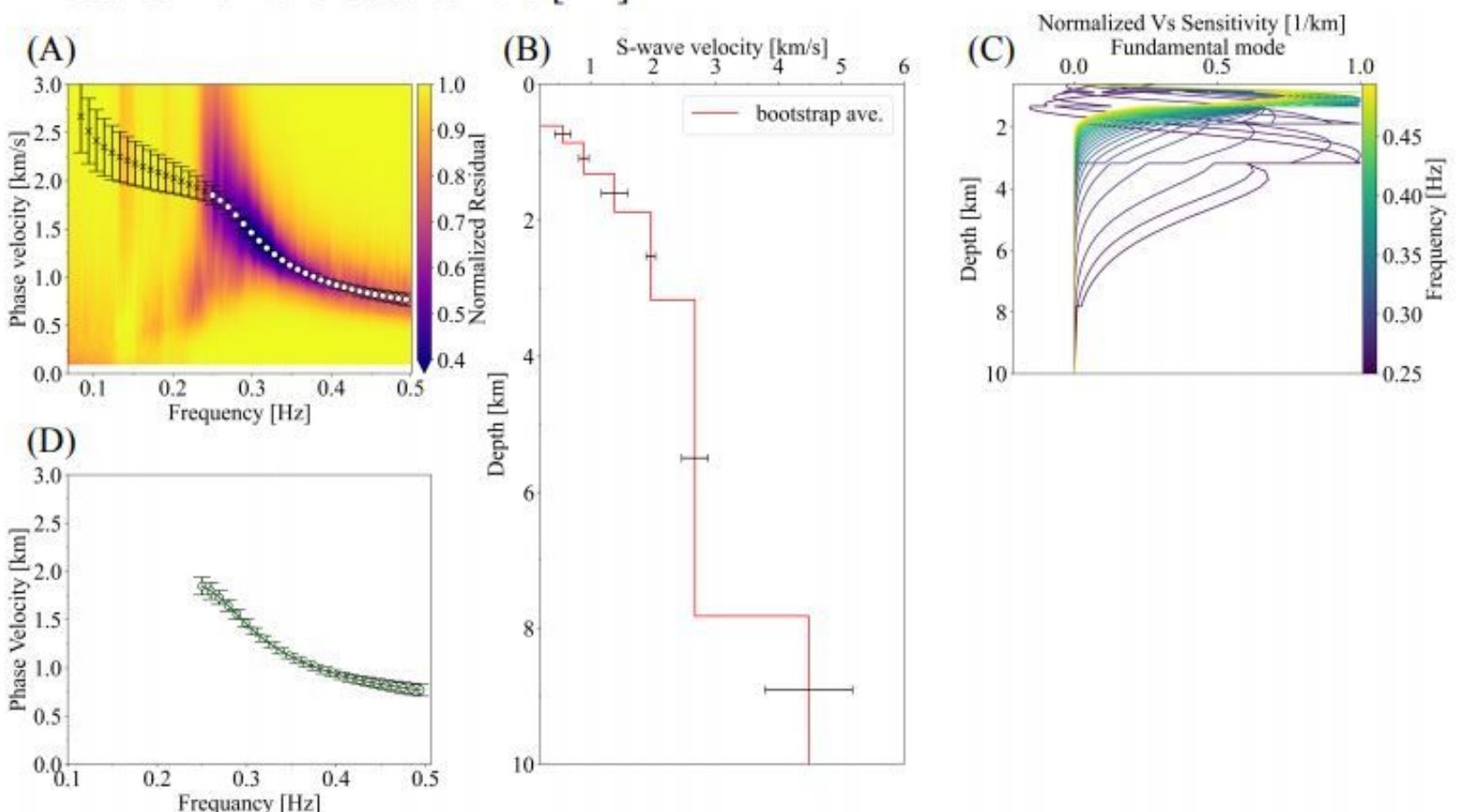

Distance from the coast : $67.48[\mathrm{~km}]$

(E)

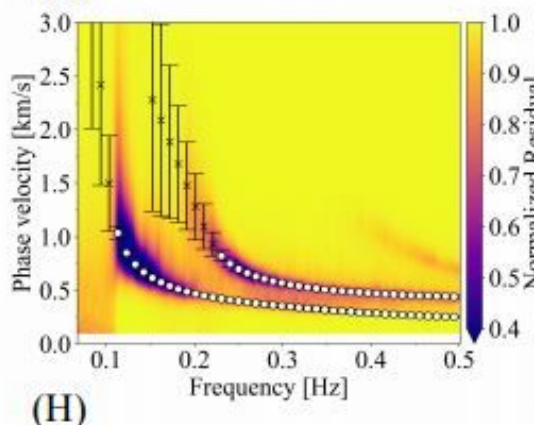

(H)

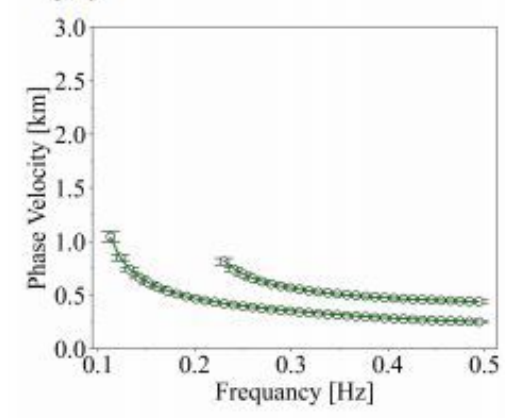

(F) , S-wave velocity $\left[\mathrm{km}_{4} / \mathrm{s}\right] 5$

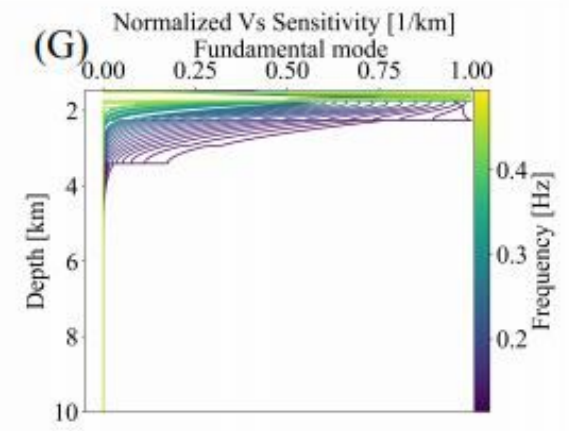

(I) Normalized Vs Sensitivity [1/km]

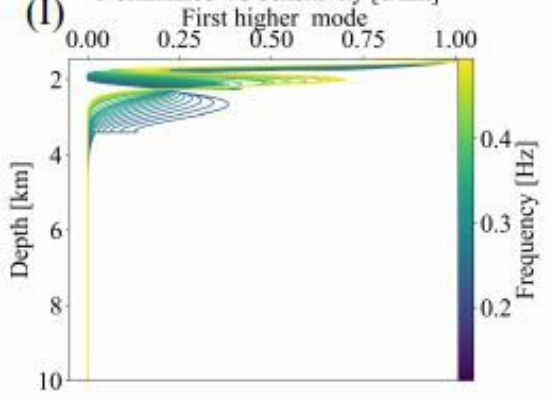

\section{Figure 3}

Short title: Phase velocity, Vs structures, and sensitivity kernel.

\section{Detailed legend:}


(A) and $(E)$ - Normalize residuals and estimation phase velocity at subarrays from the coast of 32.43 and $67.68 \mathrm{~km}$, respectively. The error bars represent the uncertainties estimated by the bootstrap method. The white dots and cross makers indicate errors less than and more than $0.1 \mathrm{~km} / \mathrm{s}$, respectively. We used phase velocity only with an error less than $0.1 \mathrm{~km} / \mathrm{s}$ for our inversion analysis. (B) and (F) - Vs structures at subarrays from the coast of 32.43 and $67.68 \mathrm{~km}$. (D) and $(\mathrm{H})$ - The white dots and green lines indicate the observed and estimated phase velocities. The errors were estimated by the bootstrap method. (C) The normalized $V$ s sensitivity kernel of the fundamental Rayleigh wave at $32.43 \mathrm{~km}$ from the coast. (G) and $(\mathrm{I})$ - The normalized $V$ s sensitivity kernels of fundamental and first higher mode Rayleigh waves at $67.68 \mathrm{~km}$ from the coast.

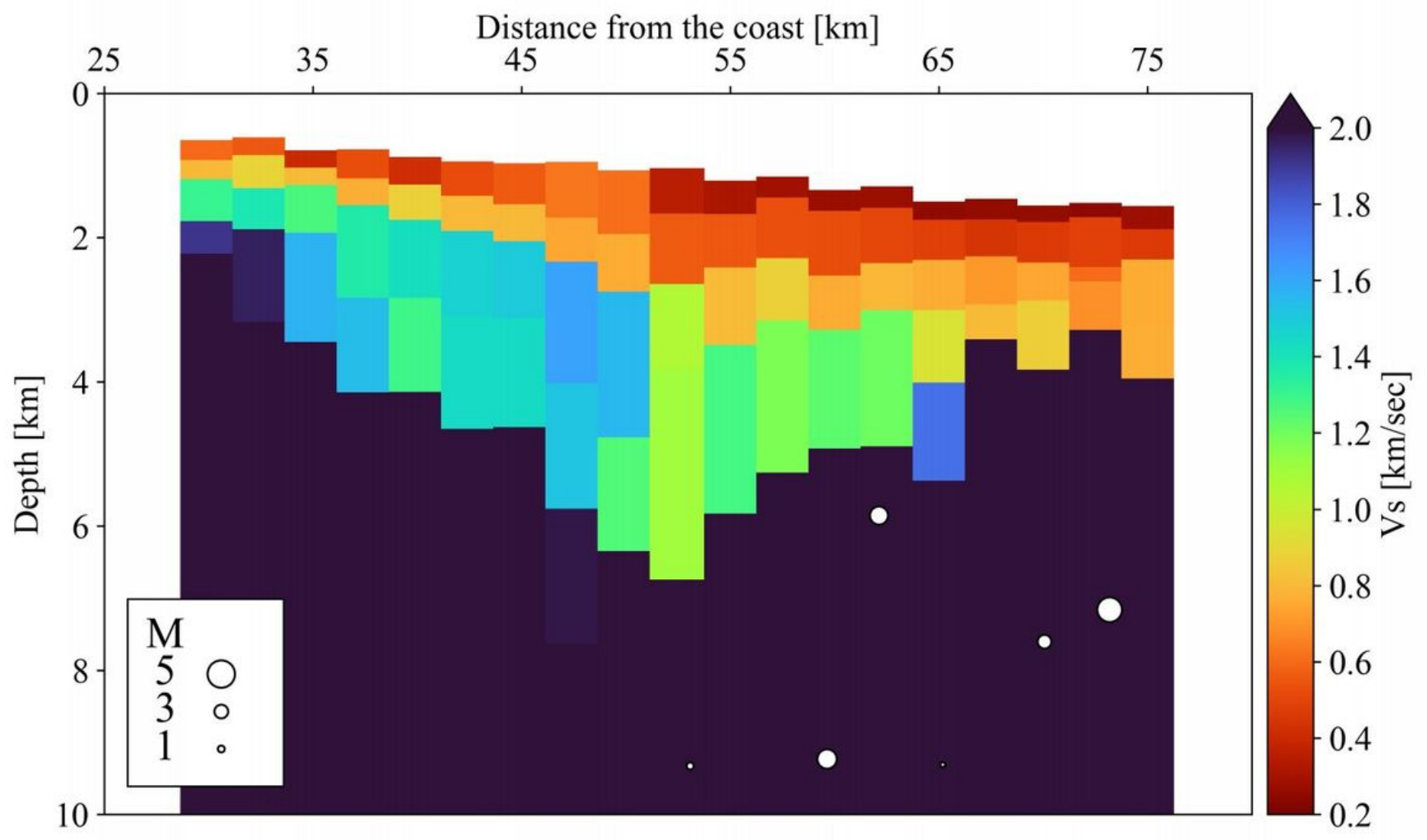

Figure 4

Short title: 2-D Vs structure estimated from phase velocity information.

\section{Detailed legend:}

2-D Vs structure estimated from phase velocity information. The white circles indicate the aftershocks off the Pacific coast of the Tohoku Earthquake determined by pop-up OBSs (Shinohara et al., 2012). The aftershocks within a box of $40 \mathrm{~km}$ in width on both sides along the fiber cable are projected onto this profile. 


\section{Supplementary Files}

This is a list of supplementary files associated with this preprint. Click to download.

- Additionalfile1.pdf

- GraphicalAbstract.jpeg 